\title{
LOS RELATOS DE MUJERES VIAJERAS ¿UNA MIRADA CRÍTICA SOBRE EL COLONIALISMO? ISABELLE EBERHARDT (1877-1904)
}

\author{
M. DOLORS GARCIA-RAMON ${ }^{1}$ \\ ABEL ALBET I MAS ${ }^{2}$
}

\begin{abstract}
Resumen: Edward Said planteó acertadamente en 1978 que Oriente no existe sino que es una construcción europea que elabora una imagen del Otro coloque no sólo define a ese Otro sino que le permite identificarse como Occidental. Pero el esquema de Said obvia la heterogeneidad del discurso colonial y oculta el rol jugado por las mujeres en el proceso de colonización. En cambio los recientes estudios feministas sobre colonialismo se centran en el papel que las mujeres europeas jugaron en el proceso colonial y exploran su complicidad tanto con el colonialismo como con su oposición a éste. La vida y obra de Isabelle Eberhardt que estuvo viajando por nuestro Oriente más próximo, el Norte de Africa, ofrecen nuevas perspectivas en torno al papel ambivalente de las mujeres en el proceso colonial y cuestionan abiertamente la noción de simple
\end{abstract} Alteridad tal y como es presentada en la obra de Said.

Palabras clave: Discurso colonial, relato de viaje, colonialismo, orientalismo y género, estudios feministas, alteridad, Isabelle Eberhardt.

\footnotetext{
Abstract: Women's Travel Narratives. A Critical GaZe on Colonialism? Isabelle EBERHARDT (1877-1904) - The aim of this paper is to analyse the life and works of a woman travel writer, Isabelle Eberhardt and to set it within the context of the recent scholarship related to travel narrative, orientalism and gender. In effect, in his important work on orientalism, Said argued that the Orient is an European construct serving an image of the Other that not only defines the Other but also identifies the self as Western. Nevertheless, Said's contribution neglects the heterogenity of colonial discourse and conceals the roles played by women in the colonization process as well as in its representation. On the contrary, recent feminist scholarship focus on the role of European women as cultural agents in the formation of imperial relations and explores women's complicity with colonialism as well as their resistance to it. But most of the research on women travellers has been carried out by English-speaking authors on English-speaking women travellers. This paper, then, tries to contribute to this field by recovering a marginal voice coming from a different place: Isabelle Eberhardt was born in Geneva (although she was of Russian origin), travelled to Tunisia and Algeria and wrote in French. The complex dynamics of complicity

1 Catedrática de Análisis Geográfico Regional; Departament de Geografia, Universitat Autònoma de Barcelona; 08193 Bellaterra (España).

Tel.: 34-935811514; Fax.: 34-935812001; E-mail: ilge8@cc.uab.es

2 Profesor Titular de Geografía Humana; Departament de Geografia, Universitat Autònoma de Barcelona; 08193 Bellaterra (España).

Tel.: 34-935811577; Fax: 34-935812001; E-mail: abel.albet@uab.es
} 
and resistance in Western women is very clear in the case of Isabelle. On one hand, she has the reputation of being "an enemy of France" but, on the other, she is central to the colonial encounter. Isabelle transgresses European norms of gender and civilization by dressing as an Arab and embracing Islam, but her self-exploration was made possible by French colonial power, and in the end, she became a player in French imperial politics. The ambivalence towards colonialism that we can observe in Isabelle's life and works (as in many other women travellers) openly questions the notion of simple Otherness as presented in Said's work. The intersection of colonial and gender discourses involves a shifting subject positioning, whereby Western women can simultaneously constitute centre and periphery, identity and alterity. Therefore, this paper hopefully shows that for the study of colonialism it is important to focus on narratives coming from the margins as they provide new perspectives which can destabilize established conceptions of the colonial relationship.

Key-words: Colonial discourse, marginal travel narratives, colonialism, orientalism and gender, feminist studies, Isabelle Eberhardt.

Résumé: Les Recits de Voyage de Femmes. Un Regard CRITIQUe SUR LE COLONIALISME? ISABELLE EBERHARDT (1877-1904) - L'objet de cet article est de présenter la vie et l'œuvre d'une femme voyageuse et écrivain, Isabelle Eberhardt et de les insérer dans les dernières recherches concernant les récits de voyage, l'orientalisme et les études de genre. Dans son important travail sur l'orientalisme, Said disait que l'Orient est une construction européenne qui offre une image de l'Autre qui ne définit pas seulement l'Autre, mais qui permet aussi de s'identifier soi-même comme Occidental. Cependant, la contribution de Said oublie l'hétérogénéité du discours colonial et cache le rôle joué par la femme dans le processus de colonisation et dans sa répresentation. Tout au contraire, les recherches féministes récentes envisagent les rôles de la femme européenne comme ceux d'agents culturels dans la formation des relations impériales et explorent la complicité des femmes avec le colonialisme et, en même temps, avec sa résistance. Mais la plupart de la recherche sur les femmes voyageuses a été faite par des auteurs anglo-américains et sur des femmes voyageuses angloaméricaines. Cet article se veut une contribution à ces recherches à travers l'étude d'une voie marginale venant d'un lieu différent: Isabelle Eberhardt est née à Genève (mais ses parents étaient d'origine russe), a voyagé à travers la Tunisie et l'Algérie et a écrit en français. La dynamique complexe de complicité et de résistance dans la femme occidentale est très évidente dans le cas d'Isabelle. D'un côté, elle a la réputation d'être "une ennemie de la France" mais, de l'autre, son rôle est central dans la rencontre coloniale. Isabelle transgresse les normes européennes de genre et civilisation en s'habillant comme une arabe et en embrassant l'Islam, mais son explorationne fut possible que grâce au pouvoir colonial français, et finalement, elle devint un acteur de la politique impériale française. L'ambivalence envers le colonialisme qu'on peut observer dans la vie et l'œuvre d'Isabelle (de la même façon que chez d'autres femmes voyageuses) met ouvertement en question la notion d'Altérité présentée dans le livre de Said. L'intersection des discours colonial et de genre conduit à un changement des positionnements, où la femme occidentale peut être considerée, en même temps, centre et périphérie, identité et alterité. Avec cet article nous voulons montrer que pour l'étude du colonialisme il est important de considérer les narratives marginales parce qu'elles fournissent des perspectives nouvelles qui peuvent mettre en question les conceptions généralement admises sur les relations coloniales.

Mots-clés: Discours coloniaux, narrations de voyage marginales, colonialisme, orientalisme et 
genre, études féministes, Isabelle Eberhardt.

Resumo: VIDA E OBRA DE UMA VIAJANTE ESCRITORA: ISABELLE EBERHARDT (1877-1904) - O objectivo deste artigo é analisar a vida e obra de uma mulher viajante e escritora, Isabelle Eberhardt, e situá-la no contexto das últimas pesquisas sobre relatos de viagens, orientalismo e estudos de género. No importante trabalho de Said sobre o orientalismo, ele dizia que o Oriente é uma construção europeia que oferece uma imagem do Outro que não só define esse Outro, mas que permite também ao próprio identificar-se como Ocidental. No entanto, a contribuição de Said esquece a heterogeneidade do discurso colonial e esconde o papel da mulher no processo de colonização e na sua representação. Pelo contrário, pesquisas recentes, feitas por mulheres, encaram o papel da mulher Ocidental como agente cultural no estabelecimento de relações de poder e exploram a cumplicidade das mulheres no colonialismo e também na resistência a esse colonialismo. Mas a maior parte da pesquisa sobre mulheres viajantes foi feita por autores de língua inglesa, sobre viajantes anglo-americanas. Este texto procura ser um contributo para estas pesquisas, através do estudo de uma voz marginal, proveniente de um lugar diferente: Isabelle Eberhardt, nascida em Genève (embora com pais de origem russa), que viajou pela Tunísia e Argélia e escreveu em francês. A complexa dinâmica de cumplicidade e resistência da mulher ocidental é muito evidente no caso de Isabelle Eberhardt. Por um lado ela tem a reputação de ser "uma inimiga de França"; por outro, o seu papel no encontro colonial é crucial. Isabelle transgride as normas europeias de género e civilização, vestindo-se como uma árabe e abraçando o islamismo, embora a sua exploração tenha sido possível graças ao poder colonial francês. No fim, ela acaba por tomar parte na política imperialista francesa. A ambivalência face ao colonialismo que se observa na vida e obra de Isabelle (tal como na de outras mulheres viajantes) põe claramente em causa a noção de alteridade apresentada no livro de Said. A intersecção do discurso colonial e de género conduz a uma mudança de posições, podendo a mulher ocidental ser considerada, ao mesmo tempo, centro e periferia, identidade e alteridade. Com este artigo procura-se mostrar que para o estudo do colonialismo é importante ter em conta narrativas marginais, pois elas podem pôr em causa concepções enraizadas sobre as relações coloniais.

Palavras-chave: Discurso colonial, narrativas de viagem marginais, colonialismo, orientalismo e género, estudos feministas, Isabelle Eberhardt.

\section{INTRODUCCIÓN}

El objetivo de este artículo es analizar la vida y obra de una escritora viajera, Isabelle Eberhardt, a la luz de los recientes estudios sobre libros de viajes, orientalismo y género. Los recientes debates feministas en torno a los estudios coloniales ponen énfasis en el papel de las mujeres en el proceso colonial y exploran su ambivalencia respecto a éste. Pero la mayoría de las investigaciones las han realizado autoras de lengua inglesa y sobre viajeras del mismo ámbito cultural. Por lo tanto, este artículo pretende hacer una contribución a este tema recuperando una voz marginal que proviene de un contexto muy diferente: Isabelle Eberhardt nació en Ginebra, aunque de padres rusos, viajó por Argelia y Túnez y escribió su obra en francés. El caso de Isabelle ilus- 
tra claramente la ambivalencia hacia la cuestión colonial que muchas viajeras experimentaron y nos ofrece nuevas perspectivas para replantear concepciones preestablecidas en el discurso colonial hegemónico.

\section{COLONIALISMO Y ORIENTALISMO FRENTE A LOS RECIENTES DEBATES FEMINISTAS}

Desde la década de los 70 se ha venido observando un renovado interés en los estudios coloniales así como en los relatos de viajes, si bien a menudo marginando el papel de la mujer occidental en ellos. No obstante, las más recientes investigaciones feministas y postcolonialistas han examinado el rol de la mujer blanca en el marco del imperialismo analizando su complejidad y cuestionando ciertas implicaciones sobre el discurso colonial hegemónico. En este sentido cabe recordar a Edward Said que, en su obra esencial Orientalismo (1978), planteó el hecho que "Oriente" no existe sino que se trata de una elucubración producida por la mentalidad europea para construir una imagen del "Otro" que no sólo defina a ese Otro sino que también permita identificarse a uno mismo en tanto que Occidental. Pero cabe destacar que el esquema de análisis que propuso Said por una parte obviaba la heterogeneidad del discurso colonial dado que establecía una clara dicotomía entre el "yo" colonizador y el "otro" colonizado y, por otra parte, ocultaba el rol jugado por las mujeres en el proceso de colonización (BLUNT et al., 1994).

Los recientes debates feministas en torno a los estudios coloniales enfatizan el concepto de ambivalencia y exploran la complicidad de las mujeres tanto con el colonialismo como con su oposición a éste, focalizando en el papel de las mujeres europeas en tanto que agentes culturales en la formación de las relaciones imperiales (MILLS, 1996). En este sentido, se alienta a escuchar la multiplicidad de voces y comprensiones diversas acerca de la colonización y el imperialismo, representadas por unas pocas mujeres que utilizaron de manera implícita (y a veces explícita) la fuerza de los componentes de raza y clase para negociar sus propios planteamientos acerca del espacio colonial (ALBET et al., 1999).

Verdaderamente, el colonialismo y su justificación ideológica ejemplificada en los libros de viajes, fueron elementos cruciales para la naciente geografía de finales del siglo XIX y principios del XX, ofreciendo una sólida base para su institucionalización como ciencia (DRIVER, 1992). Los libros de viajes sobre países exóticos (a menudo colonizados) sirven también como fuentes a través de los cuales pueden explorarse los lazos entre geografía y colonialismo y desarrollarse, de forma crítica, geografías de la alteridad. En el marco de la geografía, el componente de género ha sido un factor cada vez más esencial dentro de estos estudios, a los que este artículo pretende contribuir (DOMOSH, 1991).

LOS RELATOS DE MUJERES VIAJERAS: LA DIFERENCIA QUE APORTA EL GÉNERO

En relación a la cuestión de qué diferencia aporta el género a los libros de viajes, 
nuestra opinión es que a pesar de que los textos escritos por mujeres no son esencialmente distintos, habitualmente muestran algunos rasgos diferenciales suficientemente significativos como para ser analizados y que pueden contribuir con una nueva perspectiva a la mirada orientalista y colonialista. Una buena parte de estas diferencias radican en el status de las mujeres y sus distintos procesos de socialización en el contexto de nuestras sociedades así como, en consecuencia, en las características de los viajes que emprenden.

Una asunción tácita en la investigación feminista es que las mujeres, ellas mismas colonizadas por el género, puedan reconocer y oponerse a la colonización sintiéndose así más cercanas a las reivindicaciones de las poblaciones autóctonas. No obstante, la intersección entre género y clase abre camino a una gran complejidad de posicionamientos y actitudes. Así, en el contexto colonial, las mujeres pueden compartir (juntamente con los hombres) los discursos coloniales de poder y autoridad. Pero a menudo las mujeres viajeras se veían en la encrucijada de tener que identificarse con su raza o con su género, convirtiéndose ello en una de las fuentes de profunda ambigüedad experimentada por muchas de ellas (GARCIA RAMON et al., 1998). A pesar de ello, esta ambivalencia, a veces muy cercana a la marginalidad, fue a menudo percibida por las mujeres como generadora de fortaleza, capacitándolas para que su voz distintiva fuera escuchada. Las mujeres no estaban fuera del proyecto colonial pero sus encuentros con el colonialismo tuvieron lugar en los márgenes de dicho proyecto. Así pues, no sorprende que habitualmente las mujeres fueran más críticas con las políticas coloniales y que se preocupasen menos que los hombres de la supuesta "misión civilizadora" de Europa (McEWAN, 1996).

Los discursos de diferencia patriarcal y colonial eran espacialmente distintos. Está claro que la mujer occidental estaba marginada dentro del espacio patriarcal (en su país de origen) en el que era considerada, sobre todo, en términos de inferioridad de género. Sin embargo, en el marco del espacio colonial, las construcciones de superioridad racial y cultural podían superar dicha inferioridad ya que la mujer podía compartir con el hombre el discurso colonial de poder y autoridad. Si bien es cierto que los viajes ofrecían a la mujer la oportunidad y la libertad de moverse, espacial y socialmente, más allá de las limitaciones domésticas de sus sociedades patriarcales de origen, también es verdad que estas transgresiones sociales eran posibles en tanto que tenían lugar lejos de su hogar y de su país y sin cuestionar, pues, las construcciones patriarcales de subordinación. 
El marco colonial el proceso de exploración resulta extremadamente ambiguo; el explorador es alguien ajeno y, a la vez, partícipe, observa pero también interviene en las vidas y los territorios que visita. Habitualmente los hombres ignoraron estos conflictos de ambigüedad, sin darse cuenta de la precariedad de este posicionamiento, pero quizá las mujeres estaban más capacitadas para ello: en sus países de origen, ellas mismas eran copartícipes e intervenían en su cultura pero eran ajenas a las estructuras de poder. En las colonias se hace evidente la inconsistencia de esta postura de manera que la mujer acepta y se acomoda fácilmente a un nuevo doble papel como partícipe y forastera (MONOSH, 1991).

El caso de Isabelle Eberhardt ilustra a la perfección la ambivalencia (complicidad a la vez que resistencia) frente al colonialismo que muchas mujeres viajeras experimentaron en el espacio colonial (CARDONNE-ARLYCK, 1991).

\section{ISABELLE EBERHARDT, LA "NÓMADA APASIONADA"}

Retrato de una leyenda

Isabelle nació en Ginebra (Suiza) en 1877. Su madre, casada con un general perteneciente a la aristocracia rusa, huyó a Suiza en 1872 con el tutor de sus hijos, un anarquista ruso que había sido sacerdote ortodoxo; él fue el padre de Isabelle y quien dirigió su educación inculcándole el inconformismo que marcaría toda su vida. Fue también él quien la alentó a que usara ropa masculina, le enseñó a montar a caballo y le dio clases de árabe. En 1897 ella y su madre partieron hacia la ciudad argelina de Annaba (en aquellos momentos conocida como Bône) donde ambas se convirtieron al Islam. Isabelle pronto se sintió muy cercana a los musulmanes y empezó a escribir una series de relatos breves para la revista L'Athénée, mostrando imágenes de la vida local (BEHDAD, 1994). Su madre murió a los seis meses de llegar, circunstancia que marcó el principio de la vida nómada de Isabelle. Vestida como un hombre árabe y usando su nuevo nombre, Si Mahmoud, adquirió un caballo con el que se dirigió al Sáhara. Sin embargo en 1899 tuvo que volver a Ginebra para obtener dinero. En esa misma época murió su padre y, por diversas razones legales, Isabelle perdió su herencia, viviendo el resto de su existencia en la más absoluta pobreza.

En 1900 en El Oued encontró al hombre con el que se casaría, Slimène, furriel en los spahis (un regimiento de tropas nativas bajo mando francés). Era miembro de una orden sufí, la Qadriya, en la que Isabelle también fue iniciada. Por parte de las autoridades coloniales su presencia era vista como peligrosa para la ley y el orden, de manera que fue expulsada de Argelia un par de veces. Al casarse con Slimène, naturalizado ciudadano francés, adquirió esta nacionalidad permitiéndole regresar a Argelia. En este momento coincidiría en Argel con Barrucand, director de El Akkar, una revista bilingüe favorable a una política colonial "suave" en la que empezaría a colaborar.

En 1903 Barrucand la presentó al general Lyautey, destinado en Argelia para extender la influencia francesa hacia Marruecos; para este objetivo, Lyautey propiciaba una "penetración pacífica" más que una conquista militar de manera que muy pronto 
comprendió que el dominio que Isabelle tenía del árabe vernáculo y su amplio conocimiento de las tribus locales y de la cultura islámica la convertían en un recurso muy valioso en la obtención de información para el aparato colonial francés. Paralelamente, su casamiento con un musulmán afrancesado y su pertenencia a la Qadriya le daba acceso a lugares que ningún otro europeo osaría penetrar. A Isabelle se le propuso dirigirse al sur del distrito de Orán para informar acerca de aquellos territorios todavía desconocidos, de las tribus allí radicadas y de sus actividades militares. La proposición encajó con ella y, mientras su marido se quedaba en el norte, ella marchó al sur con un caballo, algo de dinero y el permiso del ejército francés de plena libertad de movimientos en la zona. En 1904 murió repentinamente durante una tormenta en el oasis de Aïn Sefra; su cuerpo fue encontrado dentro de su tienda y Lyautey se hizo cargo tanto de su funeral como de la búsqueda de sus manuscritos entre los escombros... que finalmente encontró.

Isabelle, que siempre tuvo grandes deseos de hacerse un nombre en el mundo de la creación literaria, publicó diversos libros (muchos de ellos editados de forma póstuma) y artículos en periódicos de París y Argel con los pseudónimos de Nicolas Podolinsky y Mahmoud Saadi. El contenido de sus escritos es básicamente intimista y, de hecho, habla poco de las mujeres (ya sean europeas o colonizadas) sobre las que ella apenas mostró ningún interés.

\section{¿Resistencia frente a las políticas coloniales?}

Isabelle fue bien conocida por sus afinidades y simpatías hacia los musulmanes y criticó abiertamente las políticas anti-árabes de la administración francesa. Por ejemplo, en Bône en 1899 cuando los estudiantes musulmanes se rebelaron contra las autoridades coloniales francesas, Isabelle estaba entre ellos. Estaba a favor de evitar una revuelta general (dado que sería fatal para los musulmanes) pero dejó escrito que "si la lucha se convierte en inevitable, no dudaré ni un sólo instante (...) quizá lucharé por los musulmanes revolucionarios tal como lo hice por los anarquistas rusos (...) aunque con más convicción y con un auténtico mayor odio contra la opresión. Me siento ahora mucho más musulmana que entonces me sentí anarquista." (citado de su Diario por KоBAK, 1989, p. 63). Es bien sabido que sus últimas actividades en la hermandad de la Qadriya, foro nativo de oposición política, no fueron muy bien vistas por los franceses. También es verdad que la mayoría de los colonizadores franceses descritos en los relatos de Isabelle son presentados como no muy inteligentes, brutales y cargados de prejuicios contra los musulmanes (Isabelle siempre despreció los colonos franceses por su vulgaridad y sus códigos sociales represivos).

Esta actitud reluctante en relación a la actuación colonizadora francesa paralelamente a sus simpatías hacia los musulmanes fueron cuidadosamente registradas en diversos informes policiales de la Argelia septentrional (llegando a provocar las citadas dos expulsiones del país). De hecho, en un momento en que la teoría de la "asimilación" era un mito operativo, los intentos extravagantes de Isabelle de mantener un "comportamiento nativo" cuestionaron seriamente dicha teoría y sugirieron que la cul- 
tura indígena tenía también sus propios méritos y virtudes y, por supuesto, ello no podía ser tolerado por los colonizadores. Pero a pesar de que las simpatías de Isabelle estuvieron siempre con los más desvalidos y de que confió románticamente en la justicia y la igualdad, nunca participó en ningún movimiento político. Su revolución siempre adquirió tintes de evasión (EBERHARDT, 1988a).

¿Una complicidad colonial, a su pesar?

A pesar de todo, cabe señalar que Isabelle siempre estuvo convencida de los beneficios esenciales de la administración francesa. Perteneció a la generación de eslavos librepensadores que siempre vieron en Francia la fuente real de todo liberalismo. Esta ambivalencia puede ayudarnos a entender algunas de sus actividades y, especialmente, las llevadas a cabo durante su último año al sur del Oranesado.

Ya en 1899, cuando pobre y errante decidió recorrer la costa sureste de Túnez en tren, acompañó durante unos meses a un agente colonial nativo. Aburrida y sin incentivos, Isabelle decidió, con notables reticencias, ayudarle a recolectar impuestos. Esta experiencia reforzaría su posicionamiento anticolonialista tal como ella misma reconoce: "Tuve una oportunidad, en el curso de mis peregrinajes por Túnez, de darme cuenta una vez más de cuan falsas y vacías son en realidad las relucientes y elegantes expresiones con las que los políticos manipulan y justifican sus propios intereses, egoísmos e intrigas (con frases como 'la acción civilizadora y pacificadora de Francia en Africa', 'la labor benéfica de la civilización ejercida sobre los pueblos indígenas de nuestras colonias')" (citado por BEHDAD, 1994, p. 120). Es cierto que esta experiencia le dio la oportunidad de ver las políticas colonialistas "en acción" pero a pesar de ello colaboró en la recolección de impuestos, de manera que su postura se mantuvo, una vez más, en la ambigüedad.

Su búsqueda de un refugio en el Sáhara la condujo a colaborar con el general Lyautey, el cual conocía muy poco de la realidad territorial de la región y los poderes fácticos de la zona (el marabú de Kenadsa, donde Isabelle deseaba ir, era especialmente poderoso); los conocimientos de Isabelle acerca del desierto y su pertenencia a la hermandad de la Qadriya, la hacían la persona ideal para ayudar al general (ERRERA, 1990; EBERHARDT, 1988b).

Los relatos que ella escribió plasman la vida en Tafilalet, en el Sáhara fronterizo con Marruecos. Describe los soldados nativos con los que viajó y con los que se identifica. También presenta la vida en los oasis de la región y las costumbres de las tribus nómadas. Se lamenta de unas formas de vida que desaparecen ante sus ojos y a menudo acusa al colonialismo de ser el responsable de ello. Pero también piensa que alguna de las políticas coloniales traerían desarrollo a estas áreas depauperadas: "para justificar nuestra presencia en el sureste de Orán, Francia tiene el imperativo deber de asegurar una paz benevolente en la zona y utilizar todo tipo de iniciativas económicas para mejorar la situación del país (...) Sin ello, la conquista de esta zona (...) será una empresa inútil que cualquier persona sensible no dudará en condenar severamente." Parece como si Isabelle ya no cuestionase la justificación global de la presencia de Francia, 
sino que sólo juzgase los medios a través de los cuales todo ello era alcanzado (EBERHARDT, 1993).

De hecho, poco a poco Isabelle fue adquiriendo una posición comprometida en relación a las políticas de Lyautey hacia las tribus y marabús y, hasta cierto punto, acabó identificándose con ellas. Quizá es oportuno recordar que los requerimientos cotidianos y de subsistencia le privaron de la libertad de optar por una visión ideológica y romántica. En un texto publicado en La Dépêche Coloniale en 1904 empleó por primera vez el pronombre "nosotros", en el sentido de "nosotros los franceses", para referirse a los movimientos de las tribus indígenas y del ejército francés, que mantenía un importante destacamento en Aïn Sefra.

Finalmente parece como si Isabelle hubiera encontrado en los planes de Lyautey un lado "humano" del colonialismo que debería traer paz y desarrollo: "nuestra mejor decisión era la de confiar esta misión al general Lyautey, cuya juventud, incomparable energía y capacidades le han permitido, en tan pocos meses, tener la situación bajo control e iniciar un plan de actuación." Es cierto que el viaje al sureste de Orán le dio al estilo de vida de Isabelle el barniz que tanto había deseado y que le había sido negado por los colonos europeos del norte, pero pagó por ello un precio muy alto, perdiendo su voz independiente. La muerte prematura de Isabelle le evitó, al menos, el dolor de constatar que la política colonial de Lyautey que ella tanto alabó culminó como tantas otras políticas coloniales para las que la paz significa simplemente intimidación.

\section{REFLEXIONES FINALES}

Los libros de viajes suponen un rico material para la comprensión de la apropiación intelectual que Europa hizo del "Oriente" en tanto que parte integrante y, a la vez, producto del vasto proceso conocido como colonialismo en el que la Geografía y los geógrafos estuvieron tan profundamente involucrados durante el periodo de institucionalización de la disciplina así como posteriormente.

El análisis de los escritos de una mujer que en los últimos años del siglo XIX estuvo viajando por nuestro "Oriente" más próximo, el Norte de Africa, ofrece nuevas perspectivas en torno al papel de las mujeres como agentes culturales en la formación de las relaciones imperiales. La ambivalencia que hemos detectado en su obra cuestiona abiertamente la noción simple de Alteridad tal y como es presentada en la obra de Said. Así, la heterogeneidad de las relaciones coloniales no es tenida en cuenta cuando existe una clara contradicción entre el "yo" colonizador y el "otro" colonizado. La intersección entre los discursos colonial y de género comporta una doble postura por parte de los sujetos implicados: Isabelle es una voz del "centro" y, a la vez, una voz de la "periferia"; fue etiquetada por los colonizadores como "una enemiga de Francia" pero el éxito de su trayectoria literaria atestiguó la complejidad del encuentro colonial.

Para el estudio del colonialismo y los libros de viajes vinculados a él, se hace patente la importancia de enfocar la cuestión a partir de narraciones "marginales" (especialmente las procedentes de autoras no anglosajonas), ya que ofrecen nuevas perspectivas que pueden replantear las concepciones preestablecidas sobre las relacio- 
nes coloniales y, al mismo tiempo, permiten ampliar el ámbito conceptual y temático de la disciplina geográfica.

\section{BIBLIOGRAFIA}

Albet, A.; M. D. GARCIA-RAMON (1999) - Reinterpretando el discurso colonial y la historia de la geografía en una perspectiva de genero. In J. NOGUE; J.L. VILLANOVA (eds.) España en Marruecos (1912-1956). Discursos geográficos e intervención territorial, Milenio, Lleida.

BEHDAD, A. (1994) - Allahou Akbar! He is a woman: colonialism, transvestism, and orientalist parasite. In A. BEHDAD. Belated travelers: orientalism in the age of colonial dissolution. Duke University Press, Durham.

CARDONNE-ARLYCK, E. (1991) - The Passionate Nomad reconsidered: an European woman in l'Algérie française (Isabelle Eberhardt, 1877-1904). In N. ChAUDURI; M. STROBEL. Western women and imperialism. Indiana University Press, Bloomington.

Domosh, M. (1991) - Towards a feminist historiography of geography. Transactions of the Institute of British Geographers, 16 (1): 95-104.

DRIVER, F. (1992) - Geography's empire: histories of geographical knowledge. Environment and Planning D: Society and Space, 10: 23-40.

EBERHARDT, I. (1988a) - Los diarios de una nómada apasionada. Mondadori, Madrid.

EBERHARDT, I. (1988b) - Écrits sur le sable. Bernard Grasset, Paris.

EBERHARDT, I. (1993) - In the shadow of Islam. Peter Owen, London.

ERRERA, E. (1990) - Isabelle Eberhardt: Cartas y diarios. Circe, Barcelona.

Garcia-RAmon, M. D.; A. Albet; J. Nogue; L. RiUdoR (1998) - Voices from the margins: gendered images of otherness in colonial Morocco. Gender, Place and Culture, 5 (3): 229-240.

KоBAK, A. (1989) - Isabelle: the life of Isabelle Eberhardt. Alfred A. Knopf, New York.

MLLS, S. (1996) - Gender and colonial space. Gender, Place and Culture, 3 (2): 125-147.

SAID, E. (1978) - Orientalism. Routledge, London. 